\title{
Why We Need a Deep Spectral Survey of the Milky Way
}

\author{
Omar M. Kurtanidze, Miriam G. Nikolashvili \\ Abastumani Astrophysical Observatory, 383762 Abastumani, Republic of \\ Georgia
}

\begin{abstract}
In this short review we briefly discuss all the extensive low dispersion objective-prism spectral surveys carried out in the last fifty years for the study of the surface and space distributions of late-type giant stars, namely $\mathrm{C}$ stars. In the light of data obtained and new discoveries, the expediency of undertaking a deep $\mathrm{m}_{I}$ low dispersion spectral survey in the near-infrared spectral region is considered.
\end{abstract}

\section{Introduction}

Theoretical models for asymptotic giant branch (AGB) stars indicate that the carbon star phase may last up to $10^{6}$ years. This lifetime is consistent with the analysis of Claussen et al. (1987), who used the space distribution of high luminosity $\mathrm{C}$ stars and their main sequence progenitors, to argue that the lifetime of $\mathrm{C}$ stars lies between $10^{5}$ and $10^{6}$ years. Theoretical aspects of the role of AGB stars in stellar and galactic evolution are discussed in detail by Jura (1991) and in IAU Coll. No. 106, Evolution of Peculiar Red Giant Stars, (eds H. Johnson \& B. Zuckerman). Interest in obtaining an accurate census of these stars using different types of surveys is stimulated by the goals of measuring their range of lifetimes in the AGB phase and the extent to which they dominate the chemical evolution of the Galaxy. Numerous spectral surveys have been done in the last fifty years. A few of them were considered and analysed by Blanco (1965, $1981)$, McCarthy $(1978,1984)$ and Mavridis (1971). Here, we concentrate on the extensive objective-prism surveys of the galactic plane and halo.

\section{Objective Prism Surveys}

Nearly one hundred and thirty years ago, Fr. Angelo Secchi reported the discovery of a new type of red star with a reversed spectrum of carbon. Starting with the pioneer work of Lee et al. (1947), low dispersion objective-prism spectra have been used for about fifty years, to identify cool red stars in our Galaxy. Nassau and Velghe (1964) introduced the method of using the near-infrared spectral range $6800-8800 \AA$ for the detection and classification of late-type stars. They also showed that carbon stars may be easily identified by their pronounced cyanogen (CN) bands at $7945 \AA, 8125 \AA$ and $8320 \AA$. 


\subsection{The Galactic Belt Surveys}

A near-infrared spectral survey of the northern part of the galactic belt was carried out at Cleveland by Nassau and Blanco (1957) to a limiting magnitude of $\sim m_{I}=10.5$. The distributions of $\mathrm{M}, \mathrm{C}$ and $\mathrm{S}$ stars have also been studied. An extension towards southern declinations was carried out by Blanco and Munch (1955).

Table 1. Low dispersion Spectral Surveys of the Milky Way

\begin{tabular}{rccccccc}
\hline No. & Observer & & & S & I & N & New \\
\hline 1 & Lee & et al. & $(1947)$ & 22,000 & 9.0 & 280 & 180 \\
2 & Nassau & et al. & $(1957)$ & 3,700 & 10.5 & 700 & 600 \\
3 & Westerlund & & $(1971)$ & 1,320 & 12.5 & 1,124 & 950 \\
4 & Alksnis & et al. & $(1989)$ & 1,200 & $14.5(\mathrm{~V})$ & & 318 \\
5 & Fuenmayor & & $(1981)$ & 650 & 13.0 & 290 & 125 \\
6 & MacConnell & & $(1988)$ & 1,200 & 13.0 & 466 & 400 \\
7 & Maehara & et al. & $(1991)$ & 1,075 & 13.0 & 749 & 200 \\
8 & Kurtanidze & et al. & $(1989 \mathrm{a})$ & 1,350 & $16.0(\mathrm{~V})$ & 1,345 & 809 \\
9 & Kurtanidze & et al. & $(1988)$ & 300 & 15.0 & 500 & 400 \\
10 & Aaronson & et al. & $(1990)$ & 400 & 15.0 & 983 & 460 \\
11 & Stephenson & & $(1989)$ & 2,400 & 14.0 & 2,100 & \\
\hline \hline
\end{tabular}

A complete deep near-infrared spectral survey for the southern Milky Way, covering the belt of $10^{\circ}$ along the galactic equator was carried out by Westerlund to a limiting magnitude of $m_{I}=12.5\left(2100 \AA \mathrm{mm}^{-1}\right.$ at A band $)$. 1124 carbon stars were identified for the longitude range from $l=235^{\circ}$ to $l=7^{\circ}$. Data on the surveys undertaken in the last twenty years are given in Table 1.

The first (yellow-red) and second (near-infrared) Abastumani surveys were carried out with the $70 \mathrm{~cm}$ meniscus telescope, equipped with a $2^{\circ}$ prism (1250 $\AA \mathrm{mm}^{-1}$ at $\mathrm{H} \gamma$ and $\sim 7000 \AA \mathrm{mm}^{-1}$ at $\mathrm{A}$ band). Kodak IIIa-J and Illa-F emulsions, hyper-sensitized in dry nitrogen, were used in the yellow-red survey for the region $l=30^{\circ}$ to $l=165^{\circ}$ and $|\mathrm{b}|<5^{\circ}$. For the spectral survey in the near-infrared, silver nitrate treated Kodak IV-N emulsion was used with a RG 695 filter for the region from $l=50^{\circ}$ to $l=115^{\circ}$ and $|b|<5^{\circ}$. Plates were exposed for $30-60 \mathrm{~m}$ and the spectra unwidened. This procedure resulted in a limiting visual magnitude of $16(\mathrm{~J}, \mathrm{~F})$ and $18(\mathrm{IV}-\mathrm{N}, \mathrm{I}=15, \mathrm{~V}-\mathrm{I}=3)$. Carbon stars were identified by the presence of cyanogen (CN) and carbon (C2) molecular bands. The main goal of survey No.9 was to reach the farthest outer parts of the Milky Way. As a result of both surveys more than $1800 \mathrm{C}$ stars were identified, with $>1200$ new ones.

\subsection{Halo Surveys}

The spectral surveys from which the list of carbon stars located at high galactic latitudes may be compiled are summarized in Table 2. Survey No.1 is a FBS undertaken by Markarian et al. (1979) in early 1963 for the identification of ultraviolet galaxies, now named as Markarian galaxies. In early 1987 we proposed 
to use this survey for the identification of carbon stars at high and intermediate galactic latitudes. During a preliminary search of plates, a few dozen faint C stars were revealed (Kurtanidze \& Nikolashvili 1989b).

Table 2. High Galactic Latitude Spectral Surveys

\begin{tabular}{lllrrrc}
\hline No. & Observer & & & S & V & N \\
\hline 1 & Markarian & et al. & $(1979)$ & 25,000 & 15.5 & \\
2 & Markarian & et al. & $(1983)$ & 1,500 & 17.5 & \\
3 & Stephenson & & $(1985)$ & 25,000 & 13.5 & 104 \\
4 & Sanduleak \& & Pesch & $(1988)$ & 1,000 & 17.0 & 32 \\
\hline \hline
\end{tabular}

The FBS and Stephenson surveys are northern all-sky surveys and contain very useful information about the distribution of carbon stars at high galactic latitudes. The SBS and Case surveys cover much smaller areas but are at least two magnitude deeper then previous surveys. They are therefore important for studying the outermost parts of the galactic halo.

Besides the regular surveys a few faint very distant carbon stars were discovered at high galactic latitudes in different pilot programs by Sanduleak (1980), Kurtanidze (1980), Sanduleak \& Pesch (1982), Ratnatunga \& Freeman (1985) and Margon et al. (1984). They are the most distant $(30<R<120 \mathrm{kpc}) \mathrm{C}$ stars known in the halo of our Galaxy.

\section{The Surface and Space Distributions}

\subsection{Surface Distribution}

Blanco (1965) studied the surface distribution of carbon stars in the galactic plane. As a result he found differences in the distribution of $\mathrm{C}$ stars in the Milky Way. These stars tend to avoid the central parts of the Galaxy whilst being preferentially located in the anti-center regions. A detailed study of the surface distribution of $\mathrm{C}$ stars in the region $30^{\circ}<1<165^{\circ}$ and $|b|<5^{\circ}$ is now finished. Of 1345 stars from our $\mathrm{J}, \mathrm{F}$ survey, 809 are new ones. The mean surface density of carbon stars is one star per square degree, i.e. it has increased by more than a factor of two (2.5) in the near-infrared. Table 3 summarizes the data for the known stars.

The statistical significance of the mean surface density increasing from the anti-center to the Cas-Cyg direction was checked using different statistical methods. It was shown that this effect is real at the 0.05 confidence level. It might be noted that the surface density in the region $30^{\circ}-50^{\circ}$ is two times lower than in nearby regions in the anti-center direction.

A pairing tendency of $\mathrm{C}$ stars at a scale of $0.2^{\circ}$ was noted by Nassau and Blanco (1954). The clustering tendency of $\mathrm{C}$ stars was later verified by Blanco and Munch (1955) and by Smith and Smith (1956). Like earlier investigators, Westerlund (1964) found a great number of carbon star pairs with separations less than $0.2^{\circ}$ using the near-infrared survey of the Milky Way. We have applied nearest neighbour statistics to single out pairs of carbon stars and to find pos- 
Table 3. Data on Survey No. 8

\begin{tabular}{lccccc}
\hline Region & New & Known & All & N/S & C/M5 \\
\hline $30^{\circ}-50^{\circ}$ & 85 & 26 & 111 & 0.56 & 0.02 \\
$50^{\circ}-70^{\circ}$ & 145 & 72 & 217 & 1.09 & 0.04 \\
$70^{\circ}-90^{\circ}$ & 156 & 108 & 264 & 1.32 & 0.06 \\
$90^{\circ}-115^{\circ}$ & 146 & 132 & 278 & 1.11 & 0.09 \\
$115^{\circ}-130^{\circ}$ & 115 & 66 & 181 & 1.12 & 0.09 \\
$130^{\circ}-145^{\circ}$ & 79 & 43 & 122 & 0.81 & 0.14 \\
$145^{\circ}-165^{\circ}$ & 83 & 89 & 172 & 0.86 & 0.25 \\
$30^{\circ}-165^{\circ}$ & 809 & 536 & 1345 & 0.99 & 0.10 \\
\hline \hline
\end{tabular}

sible members of open clusters. The observed number of $\mathrm{C}-\mathrm{C}$ and $\mathrm{C}-\mathrm{Cl}$ pairs with separations larger then $0.05^{\circ}$ are equal to the expected number, whilst the observed number of $\mathrm{C}-\mathrm{C}$ and $\mathrm{C}-\mathrm{Cl}$ pairs with separations smaller than $0.05^{\circ}$ are two times larger than expected. Nevertheless, these lie in the 0.05 confidence level interval of the Poisson distribution, i.e. there is no pronounced pairing tendency for the carbon stars in the northern Milky Way $\left(30^{\circ}<1<165^{\circ}\right)$ similar to that reported by Westerlund for the southern Milky Way. The same results were obtained by Jorgensen (1985) in an analysis of a brighter sample of $\mathrm{C}$ stars.

The importance of carbon stars as indicators of the intermediate-age stellar population is now well known. Among the results derived from the surveys carried out in the Galaxy, the Magellanic Clouds, and in dwarf spheroidal galaxies of the Local Group, it has been found that the number ratio between carbon stars and M stars of spectral type M5 or later (C/M5), is significantly correlated with the metal content of these stellar systems. The $\mathrm{C} / \mathrm{M} 5$ ratio has been determined in more than one-hundred survey fields located at latitudes of $0^{\circ}$, $+3.6^{\circ}$ and $-3.6^{\circ}$. The number of $\mathrm{M}$ stars were counted in a square belt of $2^{\circ} \mathrm{x}$ $2^{\circ}$. The results are summarized in Table 4 . It might be noted that this ratio is equal to 1.7 and 25.0 for the Large and Small Magellanic Clouds respectively.

\subsection{Space Distribution}

Westerlund (1964) computed the space densities of C stars in SA193 and found values of $15,120,7,5$ and 4 stars $\mathrm{kpc}^{-3}$ at distances of $4,6,8,10$ and $12 \mathrm{kpc}$ respectively. On the basis of his deep near-infrared survey, Fuenmayor (1981) constructed the space density functions toward the galactic center and anticenter. He found that the cool $\mathrm{C}$ stars appear to be concentrated at an average distance from the sun of $5.5 \mathrm{kpc}$ toward the center, and $0.5,5.0 \mathrm{kpc}$ toward the anti-center. The mean space density of $\mathrm{C}$ stars at these locations is 15, 20 and 50 stars $\mathrm{kpc}^{-3}$ respectively. They adopted an absolute magnitude for carbon stars of $\mathrm{M}_{I}=-5.0$. Maehara (1985) carried out the classification of fifty-nine carbon stars and calculated the space density of stars later than type $\mathrm{C} 4$. He obtained the value of $100 \mathrm{stars} \mathrm{kpc}^{-3}$.

By obtaining $K$-band magnitudes of $\mathrm{C}$ stars in the Fuenmayor anti-center sample, Jura et al. (1989) found that there is no measurable decrease in the density of $\mathrm{C}$ stars for at least $3 \mathrm{kpc}$ beyond the solar circle. Similarly, the 
density of the "very dusty" carbon stars, i.e. those with mass-loss rates greater than $2 \times 10^{-6} \mathrm{M}_{\odot} \mathrm{yr}^{-1}$, shows neither a decrease toward the anti-center nor an increase toward the center to a distance of $2.5 \mathrm{kpc}$ from the sun (Jura 1991).

The very deep survey No. 10 with subsequent observations of all detected stars in $J, H \& K$ bands and determined radial velocities will provide the main basis for the study of the space distribution of carbon stars in the Milky Way. For the local space density of $\mathrm{CH}$ stars with $-0.25>\mathrm{Mv}>-2.2$, Hartwick et al.

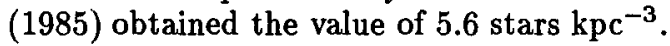

The space distribution of faint halo $\mathrm{C}$ stars was studied by Bothum et al. (1991). They showed that the density profile of halo carbon stars is very consistent with a de Vaucoleurs $r^{0.25}$ fall-off. The data cover $\sim 15$ scale radii indicating that the halo of our Galaxy may be a rather large structure. Hartwick et al. also compared the density distribution of globular clusters with that of halo stars. At large distances the profiles are in reasonable agreement whereas there are significantly more globular clusters at small radii. This is expected since the globular clusters may be strongly affected by dynamical friction.

\section{Further Prospects}

The comparative study of the distribution of the oxygen-rich and carbon-rich stars shows that the latter are much more important in the outer Galaxy than in the inner Galaxy. Therefore, to probe the farthest outer part of Galaxy in the galactic plane and halo and to study the variation of C-star characteristics with galactocentric distances, more deep low-dispersion spectral surveys must be undertaken. Such very deep surveys might be done with the largest wide field telescopes equipped with thin prisms, giving dispersions as low as $7000 \AA \mathrm{mm}^{-1}$ in the near-infrared at which late-type stars $(M, C)$ are still identifiable. For the spectral survey of the southern Milky Way, the Anglo-Australian Schmidt telescope is the best one. The limiting magnitude of $m_{I}=18$ may be reached in a $90 \mathrm{~min}$ exposure using silver-nitrate treated Kodak IV-N plates in combination with a RG 715 filter. The survey will also play an important role in the identification of different kinds of objects detected in other far-infrared surveys.

\section{Acknowledgments}

It is pleasure to thank the scientific organization committee for financial support which enabled me to attend this meeting and Sandra Harrison without whose active help it would have been impossible to attend.

\section{References}

Aaronson M., Blanco V. M., Cook K. H., \& Schechter P. L., 1989, ApJS, 70, 637

Aaronson M., Blanco V. M., Cook K. H., Olszewski E. W. \& Schechter P. L., 1990, ApJS, 73, 841

Alksne Z., Alksnis A., Ozolina V. \& Platais I., 1989, The Investigation of the Sun and Red Stars, 30, 40 
Blanco V. M. \& Munch L., 1955, Bol. Obs. Tonantznintla Tacubaya 12, 17

Blanco V. M., 1965, in Galactic Structure, A.Blaauw \& M. Schmidt, eds, (Univ. Chicago Press, Chicago)

Blanco V. M. \& McCarthy M. F. 1981, in Physical Proceses in Red Giants, I.Iben Jr. \& A. Renzini, eds

Bothum G., Elias J. H., MacAlpine G., Matthews K., Mould G., Neugebauer G. \& Reid I. N., 1991, AJ, 101, 2220

Claussen M. J., Klinmann S. G., Joyce R. R. \& Jura M., 1987, apjs, 65, 385

Dominy J. F., 1984, ApJS, 55, 27

Fuenmayor F., 1981, Rev. Mex. Astron. Astroph., 6, 83

Hartwick F. D. A. \& Cowley A. P., 1985, AJ, 90, 2244

Jura M. \& Kleinmann S. G., 1989, ApJ, 341, 359

Jura M., 1991, ARA\&A, 2, 227

Jorgensen U. G., 1985, in Cool Stars with Excesses of Heavy Elements, M. Jaschek \& P. C. Keenan, eds, p.181

Kurtanidze O. M., 1980, Astron. Circ., No. 1109, 3

Kurtanidze O. M. \& Nikolashvili M. G., 1988, Reports of the Georgian Sci. Res. Institute of the Sci. and Technical Information, No.430-G88, 1

Kurtanidze O. M. \& Nikolashvili M. G., 1989a, Astrophysics, 31, 507, references therein

Kurtanidze O. M. \& Nikolashvili M. G., 1989b, unpublished

Lee O., Gore G. \& Bartlett Th., 1947, Ann. Dearborn Observ., 5, 7 references therein

MacConnell D., 1988, AJ, 96, 354

Maehara H, 1985, PASJ, 37, 333

Maehara H. \& Soyano T. 1991, Publ. Natl. Obs. Japan, 2, 203, references therein

Margon B., Aaronson M., Leibert J. \& Maut D., 1984, AJ, 89, 274

Markarian B., Lipovetsky \& Stepanian D., 1979, Astrophysica, 16, 609

Markarian B., Lipovetsky \& Stepanian D., 1983, Astrophysica, 19, 29

Mavridis L. N., 1971, in Structure and Evolution of the Galaxy, L. N. Mavridis, ed.

McCarthy M. F. \& Blanco V. M., 1978, Mem. S. A. It., 49, 281

McCarthy M. F., 1984, in Astronomy with Schmidt-type Telescopes, M. Capaccioli, ed.

Nassau J. J. \& Blanco V. B., 1954, ApJ, 120, 464

Nassau J. J. \& Blanco V. B., 1957, ApJ, 125, 195, references therein

Nassau J. J. \& Velghe A. G., 1964, ApJ, 139, 190

Ratnatunga K. A. \& Freeman K., 1985, ApJ, 291, 260

Sanduleak N., 1980, Pub. ASP, 92, 246

Sanduleak N. \& Pesch P., 1982, Pub. ASP, 94, 690

Sanduleak N. \& Pesch P., 1988, ApJS, 66, 387

Smith E. \& Smith N., 1956, AJ, 61, 273 
Stephenson C. B., 1985, AJ, 90, 784

Stephenson C. B., 1989, Publ. Warner \& Swasey Obs., 3, 53

Westerlund B., 1964, in The Galaxy and Magellanic Clouds, Proc. IAU-URSI Symp. 20

Westerlund B., 1971, A\&AS, 4, 51 